\title{
Metformin ameliorates experimental-obesity-associated autoimmune arthritis by inducing FGF21 expression and brown adipocyte differentiation
}

\author{
Eun-Kyung Kim ${ }^{1,5}$, Seung Hoon Lee ${ }^{1,5}$, Seon-Young Lee ${ }^{1}$, Jae-kyung Kim ${ }^{1}$, Joo Yeon Jhun ${ }^{1}$, Hyun Sik Na ${ }^{1}$, \\ Se-Young Kim ${ }^{1}$, Jong Young Choi ${ }^{2}$, Chul Woo Yang ${ }^{3}$, Sung-Hwan Park ${ }^{4}$ and Mi-La Cho ${ }^{1,4}$
}

Rheumatoid arthritis (RA) is a systemic autoimmune disease involving excessive inflammation. Recently, RA associated with a metabolic disorder was revealed to be non-responsive to RA medications. Metformin has been reported to have a therapeutic effect on RA and obesity. The aim of this investigation was to study the therapeutic effect and the underlying mechanism of metformin's action in an experimental model of collagen-induced arthritis (CIA) associated with obesity. Metformin was administered daily for 13 weeks to mice with CIA that had been fed a high-fat diet. Metformin ameliorated the development of $\mathrm{CIA}$ in obese mice by reducing autoantibody expression and joint inflammation. Furthermore, metformin decreased the expression levels of pSTAT3 and pmTOR and had a small normalizing effect on the metabolic profile of obese CIA mice. In addition, metformin increased the production of PAMPK and FGF21. Metformin also induced the differentiation of brown adipose tissue (BAT), which led to a reciprocal balance between T helper (Th) 17 and regulatory $\mathrm{T}$ (Treg) cells in vitro and in vivo. These results suggest that metformin can dampen the development of $\mathrm{CIA}$ in obese mice and reduce metabolic dysfunction by inducing BAT differentiation. Thus, metformin could be a therapeutic candidate for non-responsive RA. Experimental \& Molecular Medicine (2018) 50, e432; doi:10.1038/emm.2017.245; published online 26 January 2018

\section{INTRODUCTION}

Rheumatoid arthritis (RA), a progressive and systemic form of autoimmune arthritis, is characterized by chronic inflammation and the infiltration of synovial immune cells into the affected joints. It has been well-documented that several proinflammatory cytokines are involved in the pathogenesis of RA and exacerbate its progression. ${ }^{1}$ Although there have been advances in the therapeutic functions of RA drugs, the treatment of RA remains extremely difficult., ${ }^{2,3}$ Furthermore, a significant proportion of RA patients $(30-40 \%)$ have no response to RA drugs such as anti-tumor necrosis factor (TNF)- $\alpha$ antibody therapy (such as infliximab). ${ }^{4,5}$ Currently, RA patients who show no response to RA drugs are more susceptible to non-response to other biologics used to treat RA. ${ }^{6}$ It has recently been reported that the probability of nonresponse to RA treatment is associated with metabolic disorders. ${ }^{7}$ Obesity, a metabolic disorder that plays a key role in the inflammatory response, leads to the upregulation of proinflammatory cytokine expression. It has been demonstrated that there is in increase in the expression of proinflammatory cytokines and a parallel decrease in the expression of anti-inflammatory cytokines among obese individuals compared to healthy lean individuals. ${ }^{8,9}$ Additionally, obesity is associated with inflammatory and autoimmune diseases, thus increasing the possibility of diseases such as RA occurring in obese people. ${ }^{10,11}$

Brown adipose tissue (BAT) is found predominantly in human neonates and has an important role in the modulation of body temperature. It has been demonstrated that BAT is an essential factor in non-shivering thermogenesis and energy production, and that it can therefore slow the progression of diet-induced obesity. ${ }^{12}$ The development of metabolic

\footnotetext{
${ }^{1}$ The Rheumatism Research Center, Catholic Research Institute of Medical Science, College of Medicine, The Catholic University of Korea, Seoul, South Korea; ${ }^{2}$ Division of Hepatology, Department of Internal Medicine, College of Medicine, Seoul St. Mary's Hospital, The Catholic University of Korea, Seoul, Republic of Korea; ${ }^{3}$ Division of Nephrology, Department of Internal Medicine, Seoul St. Mary's Hospital, College of Medicine, The Catholic University of Korea, Seoul, Republic of Korea and ${ }^{4}$ Divison of Rheumatology, Department of Internal Medicine, College of Medicine, Seoul St. Mary's Hospital, The Catholic University of Korea, Seoul, Republic of Korea

${ }^{5}$ These authors contributed equally to this work.

Correspondence: Professor M-L Cho, Rheumatism Research Center, Catholic Institutes of Medical Science, College of Medicine, The Catholic University of Korea, 222, Banpo-daero, Seocho-gu, Seoul 06591, Republic of Korea.
}

E-mail: iammila@catholic.ac.kr

Received 27 July 2016; revised 5 July 2017; accepted 20 July 2017 
disorders has been shown to be inhibited by BAT and several genes related to BAT differentiation, making BAT a promising candidate for the treatment of metabolic disorders. ${ }^{13}$

Fibroblast growth factor (FGF) 21 is a metabolic hormone that is primarily expressed by the liver, but is also released by adipocytes. It has been demonstrated that FGF21 has a significant role in the progression of obesity. ${ }^{14}$ Indeed, obesity is characterized by resistance to FGF $21^{15}$ and there is notable interest in the administration of FGF21 as a therapy for obesity. ${ }^{16}$ Recently, FGF 21 production was shown to be associated with BAT differentiation. The circulating level of FGF21 and the activity of BAT were increased during acute cold exposure in human subjects. ${ }^{17}$ FGF21 also demonstrated potential therapeutic function in mice with collagen-induced arthritis (CIA). ${ }^{18}$

Metformin is a biguanide anti-diabetic drug. In patients with type 2 diabetes, body weight decreases with metformin treatment. ${ }^{19,20}$ Recently, metformin has demonstrated therapeutic efficacy in other disorders. It has been suggested that metformin has the ability to ameliorate experimental autoimmune arthritis and colitis by decreasing the activation of signal transducer and activator of transcription (STAT) 3.21,22 Metformin has also been shown to have a therapeutic effect in obese mouse models via the upregulation of genes involved in BAT differentiation. ${ }^{23}$ Moreover, the expression of FGF21 was shown to increase in obese mice following treatment with metformin. ${ }^{23}$

We hypothesized that metformin would attenuate the development of CIA in mice with high-fat diet-induced obesity. The present study sought to determine whether metformin ameliorates CIA in obese mice by upregulating FGF21 expression and BAT differentiation. We measured the therapeutic and anti-inflammatory activity of metformin in obese CIA mice by studying its activity on the Th17/Treg balance and on BAT differentiation in vitro and in vivo.

\section{MATERIALS AND METHODS}

\section{Animals}

Five 7-week-old male DBA/1J mice were purchased from Orient Bio. The mice were housed in polycarbonate cages and fed $60 \mathrm{kcal}$ fatderived calories or standard mouse chow (Ralston Purina, St Louis, MO, USA) and water ad libitum. All experimental procedures were examined and approved by the Animal Research Ethics Committee of the Catholic University of Korea (permit number: CUMC:2015-0009-01), which conforms to the guidelines of the National Institutes of Health.

\section{Induction of obesity and CIA}

Chicken type II collagen (CII) immunization was performed initially when mice weighed $25 \mathrm{~g}$. Complete Freund's adjuvant (CFA; Chondrex, Redmond, WA, USA) was prepared by grinding $4 \mathrm{mg}$ of heat-killed Mycobacterium tuberculosis (H37Ra; Difco Laboratories, Detroit, MI, USA) mixing with $2 \mathrm{ml}$ of incomplete Freund's adjuvant (IFA; Chondrex). An emulsion was then formed by dissolving $4 \mathrm{mg} \mathrm{ml}^{-1} \mathrm{CII}$ (Chondrex) overnight at $4{ }^{\circ} \mathrm{C}$ in $0.5 \mathrm{M}$ acetic acid, followed by mixing the solution with an equal volume of CFA. The mice were injected intradermally in the tail. A booster injection was administered 14 days after the primary immunization. The score of arthritis severity in the joints of these mice was determined twice weekly; the arthritis score was recorded as the sum of the scores on a scale of $0-4$.

\section{Metformin and Enbrel treatment}

Metformin was obtained from Sigma-Aldrich (St Louis, MO, USA) and dissolved in saline. Mice were given $50 \mathrm{mg} \mathrm{kg}^{-1}$ of oral metformin daily for 13 weeks starting on day 7 after the first immunization. Enbrel (Pfizer, New York, NY, USA) was injected subcutaneously (SC) 3 times per week after the initial immunization. The Enbrel dose was $100 \mu \mathrm{g}$ per mouse. Control mice were injected with saline.

\section{Histological assessment}

Mouse joint, liver and interscapular BAT (BAT) samples were obtained 13 weeks after immunization and fixed in $4 \%$ paraformaldehyde, decalcified in calci-clear rapid (National diagnostics), and embedded in paraffin. The joint, liver and iBAT were sectioned at a thickness of $6 \mu \mathrm{m}$, deparaffinized using xylene, dehydrated through a gradient of alcohol and then stained with hematoxylin and eosin (H\&E), or Safranin O. The H\&E-stained sections were scored for inflammation and bone erosion. Inflammation was scored according to the following criteria: $0=$ no inflammation, $1=$ slight thickening of the lining layer or some infiltrating cells in the underlying layer, $2=$ slight thickening of the lining layer plus some infiltrating cells in the underlying layer, 3 =thickening of the lining layer, an influx of cells in the underlying layer, and the presence of cells in the synovial space, and $4=$ synovium highly infiltrated with many inflammatory cells. Cartilage damage was determined using Safranin O staining where the extent of cartilage damage was scored according to the following criteria: $0=$ no destruction, $1=$ minimal erosion limited to single spots, $2=$ slight-to-moderate erosion in a limited area, $3=$ more extensive erosion, and $4=$ general destruction. Immunohistochemistry (IHC) staining was performed using a Vectastain ABC kit (Vector Laboratories). The tissues were incubated with anti-IL-17 and antiIL-6 antibodies (Santa Cruz Biotechnology Inc., SantaCruz, CA, USA) overnight at $4{ }^{\circ} \mathrm{C}$. These primary antibodies were detected with a biotinylated secondary linking antibody for $40 \mathrm{~min}$, followed by incubation with streptavidin-peroxidase complex for $1 \mathrm{~h}$. The final color product was developed using 3,3'-diaminobenzidine (DAB) chromogen (DAKO, Carpinteria, CA, USA). Positive cells were counted, with the results expressed as the mean \pm s.d.

\section{Confocal microscopy}

For immunostaining, $7 \mu \mathrm{m}$ tissue sections of spleens were stained. To analyze the populations of $\mathrm{T}$ helper cells, we used Alexa 488 conjugated anti-CD4, PE-conjugated anti-IL-17, APC-conjugated anti-CD25, and PE-conjugated anti-Foxp3 antibodies (eBiosciences, San Diego, CA, USA). To analyze the populations of STAT, AMPK and mTOR, the samples were stained with Alexa 488 conjugated anti-CD4, PE-conjugated anti-phosphorylated STAT-3 tyrosine 705, PE-conjugated anti-phosphorylated STAT-3 tyrosine 727, anti-mTOR, anti-AMPK, and anti-FGF21 antibodies, and anti-rabbit IgG-PE secondary antibody. The nuclei were stained with 4',6-diamidino-2phenylindole. The stained sections were analyzed using a Zeiss microscope (LSM 510 Meta; Carl Zeiss, Oberkochen, Germany) at $\times 400$ magnification. Positive cells were counted, and the numbers expressed as the mean \pm s.d. 
Biochemical analyses

Blood samples were collected from all treated and control mice at 13 weeks and stored at $-70{ }^{\circ} \mathrm{C}$ until use. The levels of AST, ALT, HDL- and LDL-cholesterol were measured using commercial kits from Asan Pharmaceutical Co. (Hwangseong-gi, Gyeonggi-do, Korea).

\section{Analysis of gene expression by real-time quantitative PCR}

Total RNA was extracted using TRIzol (Molecular Research Center, Cincinnati, OH, USA). Two micrograms of total RNA was reverse transcribed using the Superscript Reverse Transcription system (Takara, Shiga, Japan). Quantitative real-time PCR (qRT-PCR) was performed with LightCycler FastStart DNAmaster SYBR green I (Takara) fluorescent dye using an ABI PCR machine. Primers for FGF-21 (forward: GCATACCCCATCCCTGACTC, reverse: ACCAC TGTTCCATCCTCC CT), IL-17 (forward: CCTCAAAGCTCAGCG TGTCC, reverse: GAGCTCACTTTTGCGCCAAG), IKBKE (forward: CCCAAAGTTCGTCCCTAAGGTTG, reverse: ATCAACGCCTGTCC ATCCAGCA) and $\beta$-actin (forward: $5^{\prime}$-GAAATCGTGCGTGACATC AAAG-3', reverse: 5'-TGTAGTTTCATGGATGCCACAG-3') were designed using Primer Express (Applied Biosystems, Foster City, CA). The mRNA expression levels were normalized to those of $\beta$-actin.

\section{Murine T-cell isolation and differentiation}

Spleen cell cultures were performed in RPMI 1640 medium supplemented with $5 \%$ FBS. To purify $\mathrm{CD}^{+}{ }^{+} \mathrm{T}$ cells, the cells were incubated with $\mathrm{CD} 4$-coated magnetic beads and isolated using magneticactivated cell sorting (MACS) separation columns (Miltenyi Biotec).
Positively selected $\mathrm{CD}^{+} \mathrm{T}$ cells were stimulated with plate-bound anti-CD3 $\left(0.5 \mu \mathrm{g} \mathrm{ml}^{-1}\right)$, soluble anti-CD28 $\left(1 \mu \mathrm{g} \mathrm{ml}^{-1}\right.$; both from BD Biosciences), anti-interferon- $\gamma\left(2 \mu \mathrm{g} \mathrm{ml}^{-1}\right)$, anti-IL-4 $\left(2 \mu \mathrm{g} \mathrm{ml}^{-1}\right)$ antibodies, recombinant TGF- $\beta$ ( $\left.2 \mathrm{ng} \mathrm{ml}^{-1}\right)$, and recombinant IL-6 $\left(20 \mathrm{ng} \mathrm{ml}^{-1}\right)$ (R\&D Systems) for 3 days to achieve polarization of Th17 cells.

\section{Co-culture experiment of Th17 cells with BAT}

Spleen Th17 cells were seeded in 24 -well plates at $1 \times 10^{6}$ per well in 5\% RPMI, and BAT was layered onto Th17 cells. Culture plates were incubated at $37^{\circ} \mathrm{C}$ for $72 \mathrm{~h}$, and then, the culture supernatants were collected.

\section{BAT transplantation}

BAT was removed from DBA1/J donor mice (age 7 weeks) and washed in sterile PBS. We then transplanted $0.2 \mathrm{~g}$ donor BAT into the subcutaneous dorsal region of CIA mice as quickly as possible. Recipient mouse spleen samples were obtained 5 weeks after the transplantation. The control group underwent the same procedure.

\section{Enzyme-linked immunosorbent assay}

The amounts of IL-17 in culture supernatants derived from mice were measured by sandwich enzyme-linked immunosorbent assay (ELISA, R\&D Systems). Alkaline phosphatase (Sigma-Aldrich) was used for color development. Absorbance was measured at $405 \mathrm{~nm}$ on an ELISA microplate reader (Molecular Devices). a

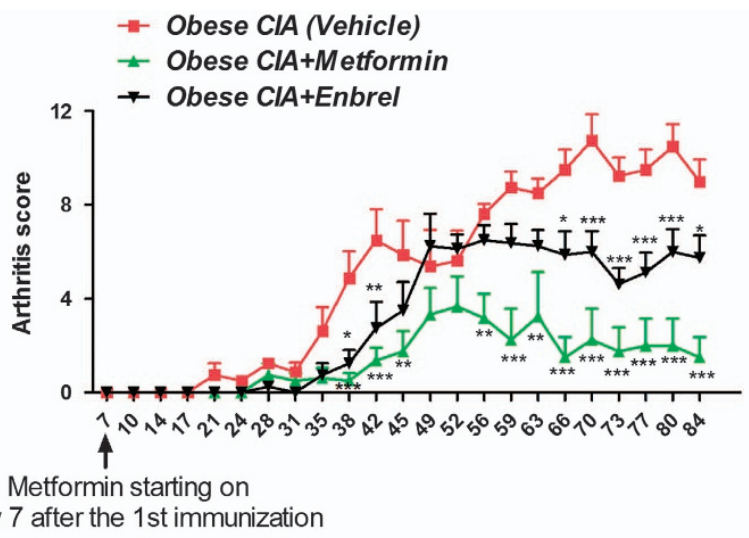

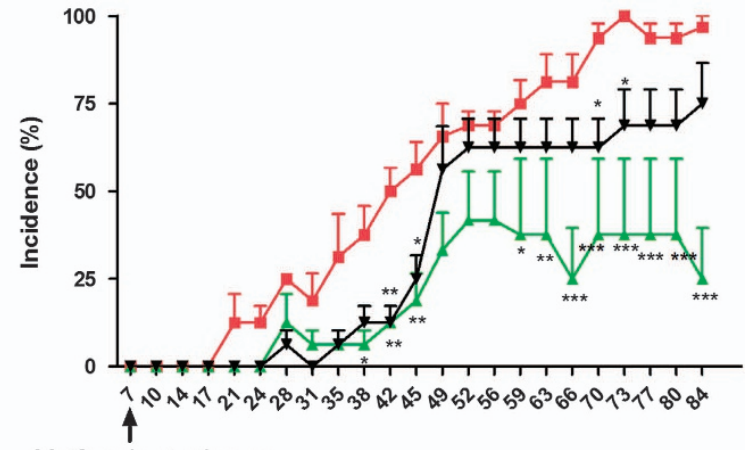

Metformin starting on

day 7 after the 1 st immunization b

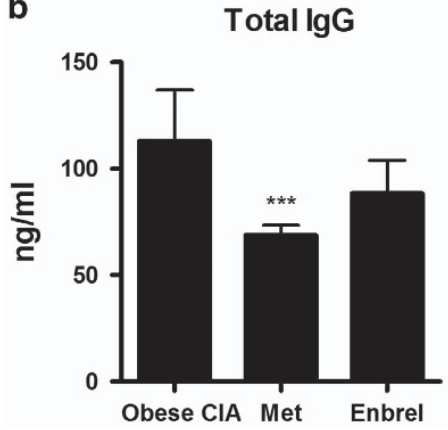

$\lg G 1$

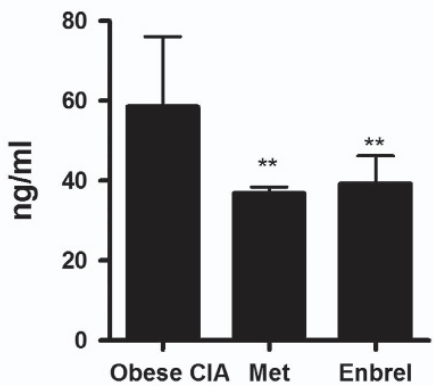

$\lg$ Ga

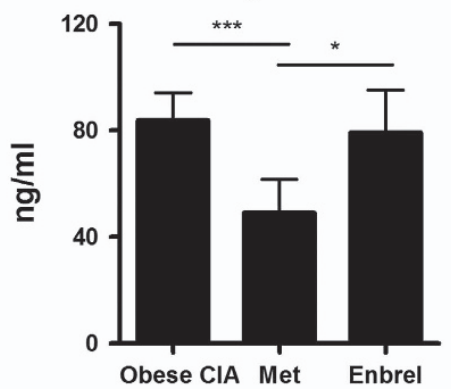

Figure 1 Treatment with metformin attenuated in obese collagen-induced arthritis (CIA) mice. (a) Reduction in the arthritis score and arthritis incidence in obese CIA mice treated with metformin. (mean \pm s.e.m. of five mice per group, ${ }^{*} P<0.05,{ }^{* *} P<0.01,{ }^{* * *} P<0.001$ ). (b) Levels of IgG, IgG1 and IgG2a were determined by enzyme-linked immunosorbent assay. (Mean \pm s.d. of five mice per group, ${ }^{*} P<0.05$.). 
$H \& E$
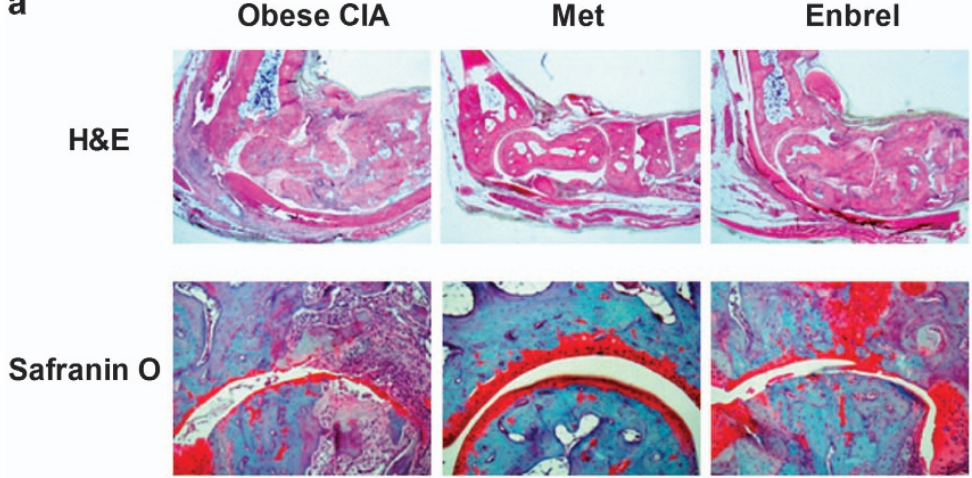

b

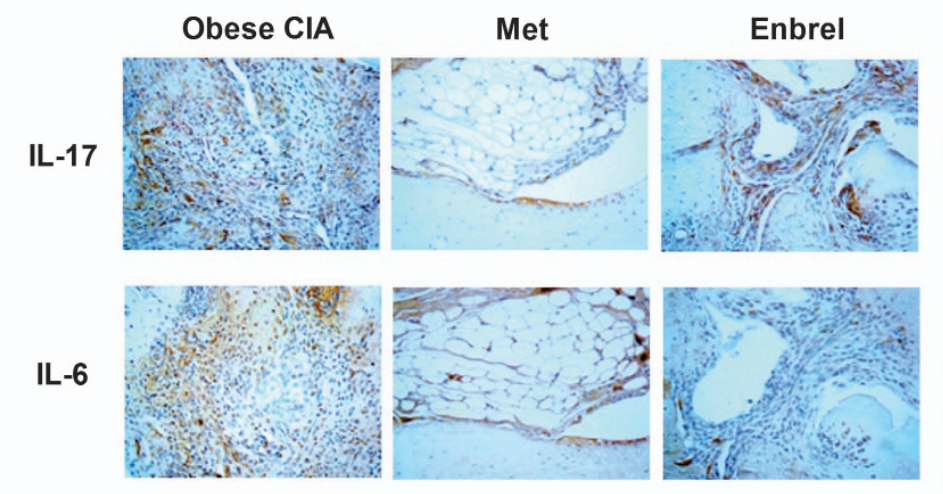

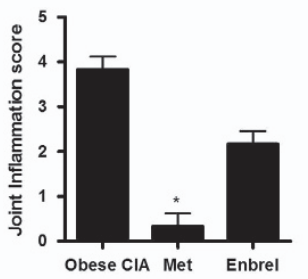
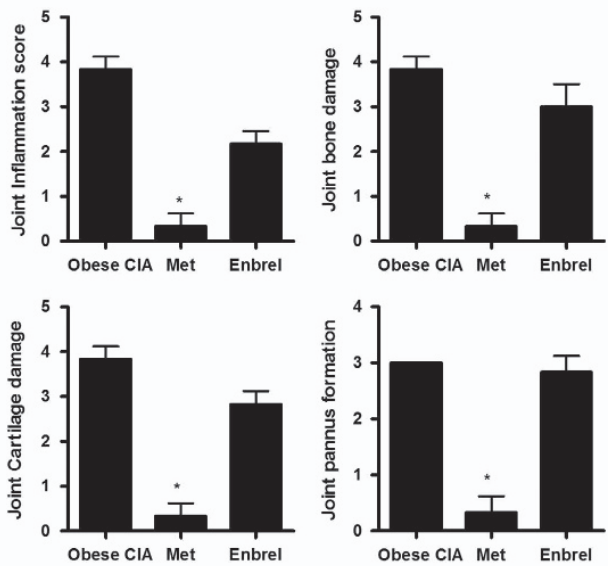
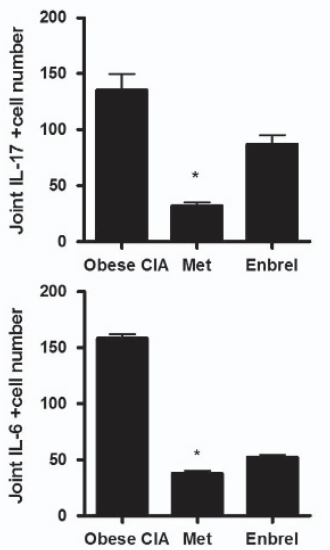

Figure 2 Effects of metformin on proinflammatory molecules in the joints of collagen-induced arthritis (CIA) and obese CIA mice. (a) Histologic features of the joints were stained with hematoxylin and eosin (H\&E), and Safranin O. (b) Tissue sections from joints were immunohistochemistry stained with anti-IL-6 and anti-IL-17 antibodies. (Values are the mean \pm s.d., ${ }^{*} P<0.05,{ }^{* *} P<0.01$.)

\section{Measurement of IgG concentrations}

Blood samples were taken from the orbital sinuses of mice, and the serum concentrations of IgG, IgG1 and IgG2a were measured using mouse IgG, IgG1 and IgG2a ELISA quantitation kits (Bethyl Laboratories), respectively.

\section{Intracellular staining and flow cytometry}

Cells were stimulated with $25 \mathrm{ng} \mathrm{ml}^{-1}$ PMA (Sigma-Aldrich, St Louis, MO), $250 \mathrm{ng} \mathrm{ml}^{-1}$ ionomycin (Sigma-Aldrich) and Golgi Stop (BD Biosciences, San Diego, CA) in $5 \% \mathrm{CO}_{2}$ at $37^{\circ} \mathrm{C}$ for $4 \mathrm{~h}$. Cells were stained with Percp-conjugated anti-CD4 antibody and APC-conjugated anti-CD25 Ab (BD Pharmingen) and then stained with APC-conjugated anti-IFN- $\gamma$, PE-conjugated anti-IL-4, FITC-conjugated anti-IL-17 or PE-conjugated anti-Foxp3 (all from eBiosciences), followed by fixation and permeabilization using the Cytofix/Cytoperm Plus Kit (BD Biosciences) according to the manufacturer's instructions. All samples were processed with FACSCalibur (BD Pharmingen), and the data were analyzed using FlowJo software (Tree Star, Ashland, OR, USA).

\section{Statistical analysis}

Statistical analyses were performed using GraphPad Prism 5 software. Differences between treatment groups were tested for statistical significance with the Mann-Whitney $U$-test. The results are expressed as the means \pm s.d. (or means \pm s.e.m.). The data were considered significantly different at $P<0.05$ (two-tailed).

\section{RESULTS}

Metformin ameliorates the progression of CIA in obese mice Compared to mice that received vehicle, metformin-treated mice had a significantly lower arthritis score during the entire experimental period. Treatment of mice with metformin also attenuated the progression of arthritis compared with mice receiving Enbrel (Figure 1a). The concentrations of total IgG, IgG1 and IgG2a in the serum of metformin-treated obese CIA mice were less than those in vehicle-treated and Enbrel-treated obese CIA mice (Figure 1b).

The anti-inflammatory profile of metformin-treated mice Consistent with the arthritis score, minimal signs of inflammation were detected in the metformin-treated obese CIA mice, whereas extensive infiltration of immune cells was observed in vehicle- and Enbrel-treated obese CIA mice. Histological analyses also showed that joint destruction, bone and cartilage damage, and pannus formation were ameliorated in the metformin-treated obese CIA mice compared to vehicle- or Enbrel-treated obese CIA mice (Figure 2a). 
a
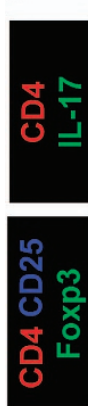

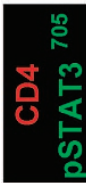
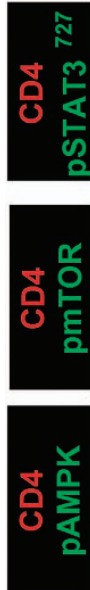
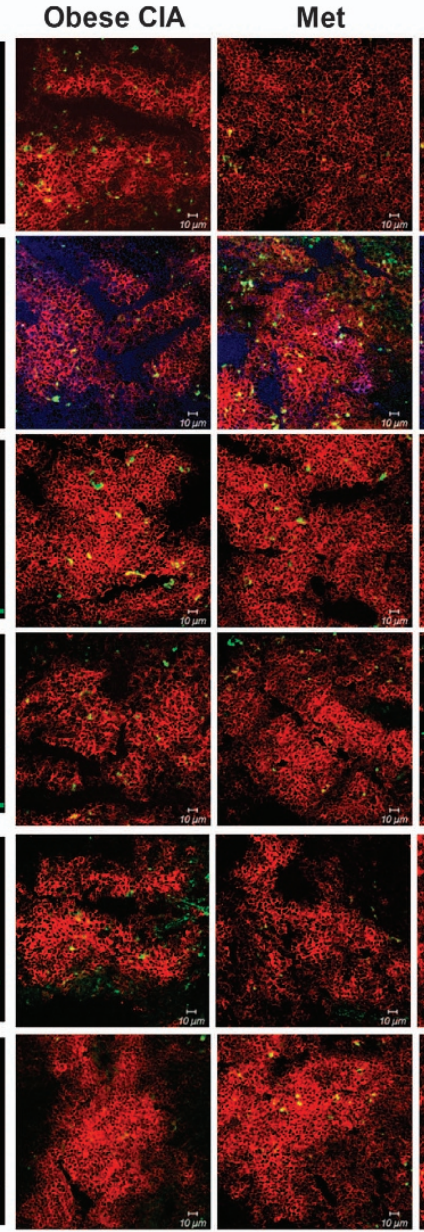

Enbrel
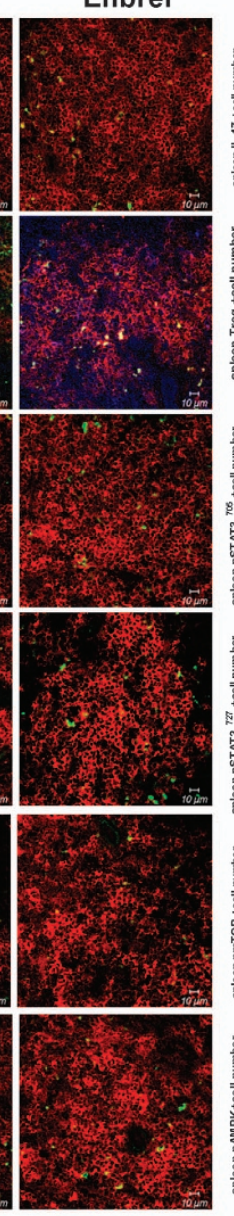
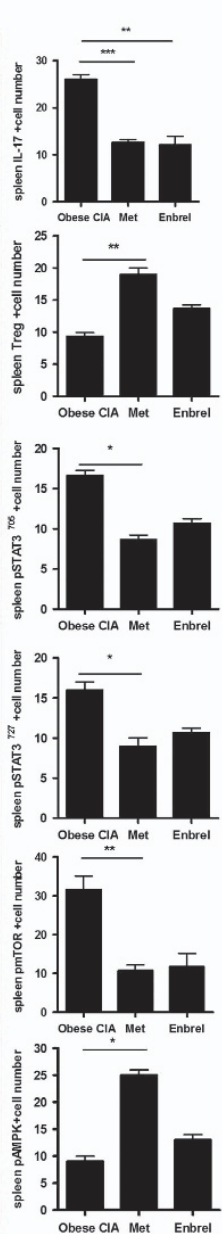

b
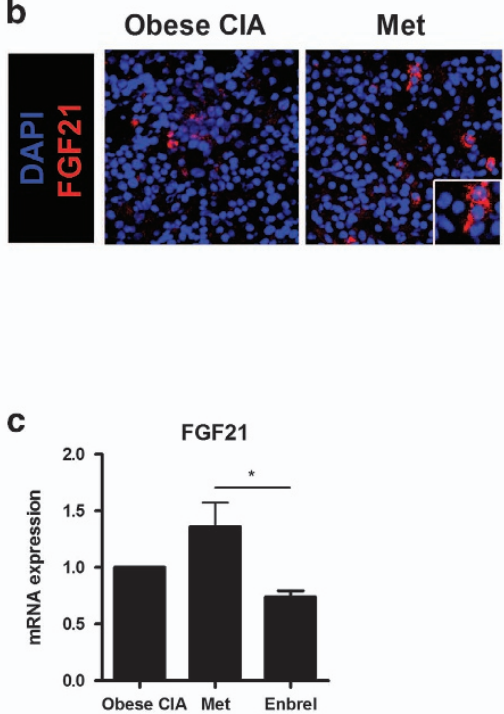

Figure 3 Metformin reduced STAT3 phosphorylation, decreased the frequency of Th17 cells within the population of CD4 ${ }^{+}$T cells and induced FGF21 expression in collagen-induced arthritis (CIA) and obese CIA mice. (a, b) Spleen tissues from each mouse were examined by immunofluorescence staining. Original magnification $\times 400$. (c) Expression of FGF21 mRNA in isolated splenocytes was measured by real-time polymerase chain reaction, with the results normalized to the expression of $\beta$-actin $m R N A$. (Values are the mean $\pm s . d$., ${ }^{*} P<0.05$, $* * P<0.01$.)

Additionally, the expression levels of IL- 6 and -17 in the joints were significantly lower in the metformin-treated obese CIA mice than in the vehicle-treated obese CIA mice (Figure 2b).

Counter-regulatory effects of metformin on Th17/Treg cells in obese CIA mice are associated with activation of STAT3 Confocal imaging showed significantly fewer $\mathrm{CD} 4^{+} \mathrm{IL}-17^{+}$, $\mathrm{CD}^{+}{ }^{+}$PSTAT3 $\mathrm{Tyr} 705^{+}, \mathrm{CD}^{+}{ }^{+}$PSTAT3 $\mathrm{Ser}_{2} 7^{+}$and $\mathrm{CD} 4^{+}$ pmTOR $^{+}$cells in the spleen tissue of the metformin-treated obese CIA mice than in that of the vehicle- or Enbrel-treated obese CIA mice. However, there were significantly more $\mathrm{CD} 4^{+}$ $\mathrm{CD}_{2} 5^{+} \mathrm{Foxp}^{+}$and $\mathrm{CD} 4^{+} \mathrm{pAMPK}^{+}$cells in the spleen tissue of metformin-treated obese CIA mice than in that of vehicle- or Enbrel-treated obese CIA mice (Figure 3a). There was also a significant upregulation of FGF $21^{+}$cells in spleen tissue from metformin-treated obese CIA mice compared to the vehicleor Enbrel-treated obese CIA mice (Figure 3b). The gene expression of FGF21 in the spleen was also significantly increased with metformin treatment (Figure 3c).

Effect of metformin treatment on metabolic profiles in obese CIA mice

Metformin treatment caused no changes in weight (data not shown) or other macroscopic changes (Figure 4a). The weight of the liver also did not change with metformin treatment (Figure 4b). However, H\&E staining showed a decrease in the immune cell infiltration of the liver among metformin-treated obese CIA mice compared to vehicle-treated obese CIA mice (Figure 4c). Metformin slightly reduced the levels of AST, ALT and LDL in the serum from obese CIA mice. The HDL level did not change with metformin treatment (Figure 4d). The levels of IL-17 and IKBKE mRNA in the liver of metformintreated obese CIA mice were significantly downregulated compared to those in the vehicle-treated obese CIA mice (Figure 4e). However, treatment with metformin significantly 
a

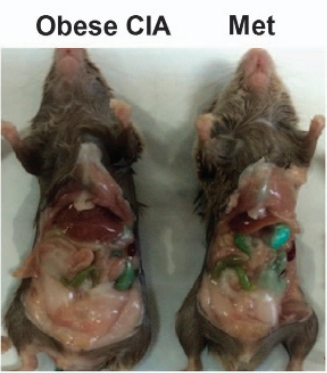

b

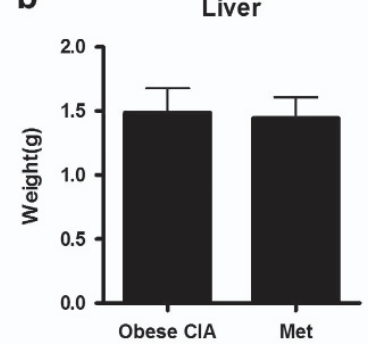

c

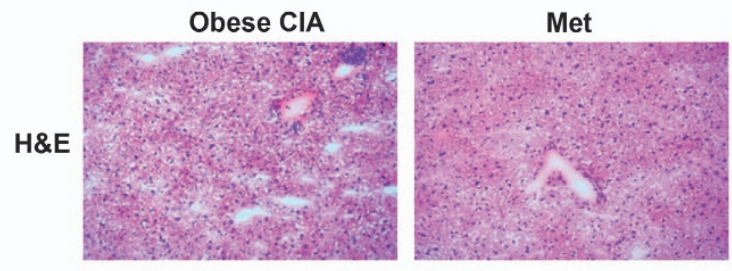

d

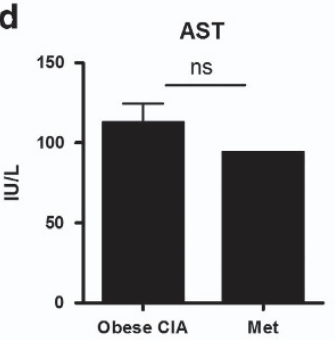

e

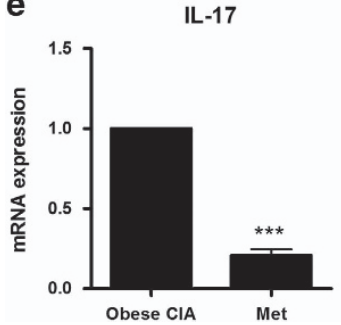

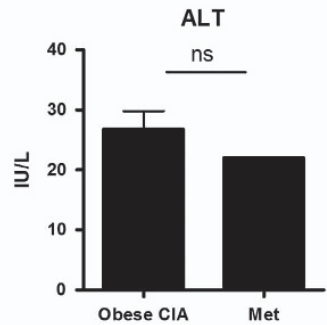
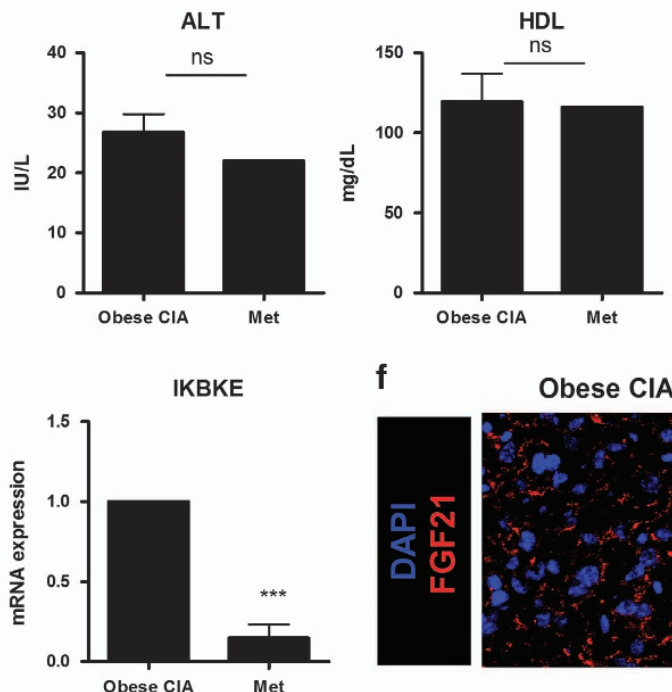

f

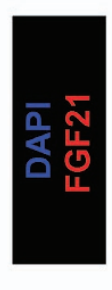

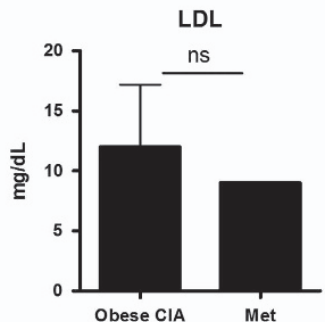

Met
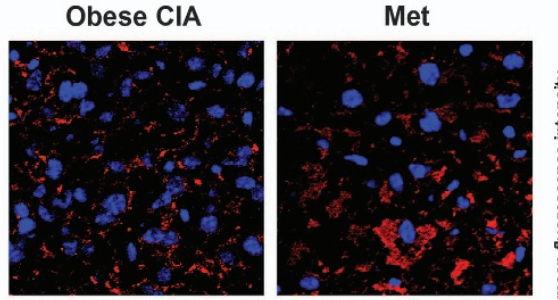

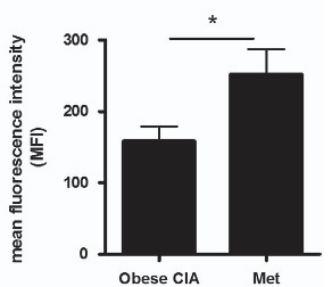

Figure 4 Metformin treatment ameliorated fatty liver and normalized metabolic profiles. (a, d) Obese collagen-induced arthritis (CIA) mice or metformin-treated obese CIA mice had a similar phenotype, but different metabolic profiles. (b) Liver tissues obtained from obese CIA and metformin-treated obese CIA mice were weighed. (c) Sections were stained with hematoxylin and eosin (h\&e). Original magnification $\times 400$. (e) Expression of IL-17 and IKBKE mRNA in isolated hepatocytes was measured by real-time polymerase chain reaction, with the results normalized to the expression of $\beta$-actin mRNA. (f) Liver tissues from obese $\mathrm{ClA}$ and metformin-treated obese $\mathrm{ClA}$ mice were examined by immunofluorescence staining. Original magnification $\times 400$. (Values are the mean \pm s.d., ${ }^{*} P<0.05$, ${ }^{* *} P<0.01$, $* * * P<0.001$.)

increased the expression of FGF21 in the liver of obese CIA mice (Figure 4f).

\section{Effect of metformin treatment on BAT regulation in obese} CIA mice

There was an increase in the weight of BAT in obese CIA mice treated with metformin, but it was not significant (Figure 5a). Metformin treatment also reduced the infiltration of immune cells in the BAT of obese CIA mice (Figure 5b). FGF21 production in the BAT of obese CIA mice was also increased with metformin treatment (Figure $5 \mathrm{c}$ ).

Effect of co-culture and transplantation with BAT on the suppression of Th17 and IL-17

IL-17 levels significantly decreased in the culture medium obtained from Th17 cells co-cultured with BAT (Figure 6a). Th1 and Th17 cell differentiation was also decreased in the spleen of mice transplanted with BAT, but the difference was not significant (Figure 6b).

\section{DISCUSSION}

Metformin is known to be an effective treatment for type 2 diabetes and obesity. Recently, metformin was revealed to have an anti-arthritic effect on experimental autoimmune arthritis. ${ }^{21}$ To the best of our knowledge, the mechanism of metformin's action and its activity on BAT in obese CIA mice has yet to be documented. In this investigation, the therapeutic functions of metformin in an obese CIA mouse model were observed, and the findings suggested the possibility of using metformin in therapeutic strategies against RA associated with obesity.

An important observation was the therapeutic efficacy of metformin in obese CIA mice and its association with the reciprocal Th17/Treg balance and the upregulation of BAT differentiation. The downregulation of Th17 and the upregulation of Tregs is an important therapeutic process in experimental autoimmune arthritis. ${ }^{21,24,25}$ A high-fat diet has been shown to induce the downregulation of BAT while increasing markers of fatty liver, including AST and ALT. ${ }^{26}$ By contrast, the upregulation of BAT has been shown to reduce the levels of plasma ALT and AST. ${ }^{27}$ It is also widely believed that BAT has therapeutic potential in the treatment of metabolic disorders. ${ }^{13}$ 
a
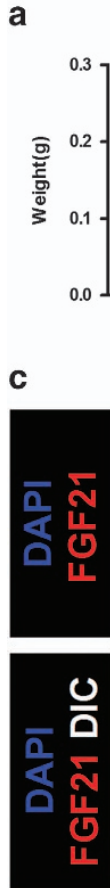

b

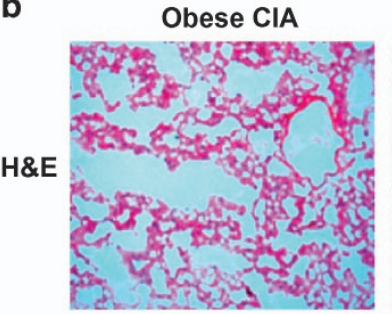

Met

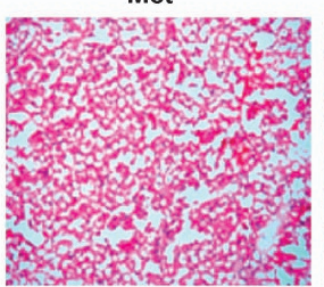

Enbrel

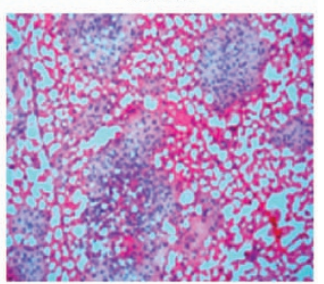

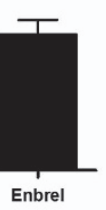
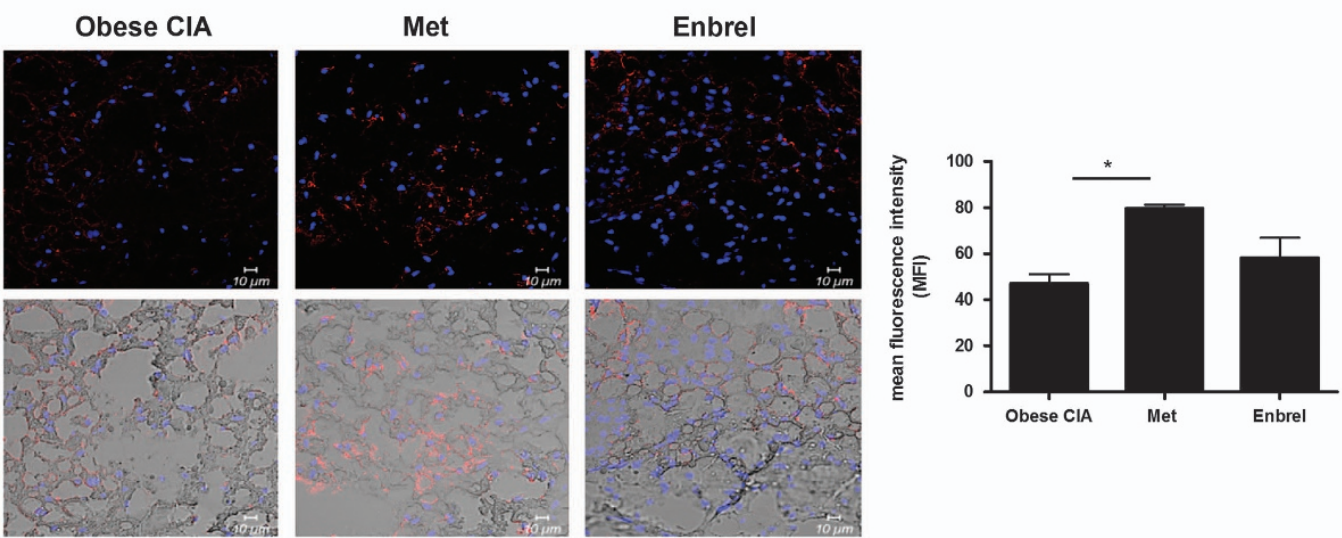

Figure 5 Brown adipose tissue was upregulated by metformin treatment. (a) Weight of brown fat obtained from obese ClA mice or metformin- or Enbrel-treated obese CIA mice. (b) Histologic features of the brown fat tissues stained with hematoxylin and eosin (h\&e). Original magnification $\times 200$. (c) Brown fat tissues from each mouse were stained for FGF $21^{+}$cells. Original magnification $\times 400$.

Here it was found that metformin reduces obesity and has an anti-arthritic effect via inducing BAT and restoring the reciprocal balance between Th17 and Tregs. In addition, metformin reduced the infiltration of immune cells in the liver and BAT. We observed that transplantation of BAT decreased Th17 cell differentiation, while a co-culture with BAT reduced IL-17 expression in vitro. These results suggest that metformin can decrease the inflammatory response in RA associated with metabolic disorders.

Metformin decreased the expression of pSTAT3 and mTOR and enhanced pAMPK expression. The inflammatory response and the expression of proinflammatory cytokines, including IL- 6 and -17 , are induced by pSTAT3. ${ }^{28}$ By contrast, AMPK inhibits the activation of mTOR, which in turn leads to enhanced expression of pSTAT3. ${ }^{29,30}$ It has been demonstrated that the IL-6 level in the synovial fluid and blood of RA patients is excessively high and is positively correlated with joint destruction. ${ }^{31,32}$ IL-17 is also produced at high levels in the synovium and synovial fluid of RA patients. ${ }^{33}$ In this study, a significant downregulation of IL- 6 and -17 was found in the joint tissue of metformin-treated obese CIA mice. Additionally, the expression levels of mTOR and pSTAT3 were reduced with metformin treatment. Since the inhibition of proinflammatory cytokines and pSTAT3 expression plays a key role in the suppression of inflammation, our results reveal that metformin has anti-inflammatory activity in obese CIA mice.

Previously, metformin was found to downregulate the levels of obesity-related factors, including cholesterol and LDL, but to promote FGF21 expression in obese mice. ${ }^{23}$ Since FGF21 has been shown to be a key regulator of obesity, ${ }^{14}$ enhancing
FGF21 expression has great potential therapeutic efficacy. FGF21 also attenuates obesity-mediated inflammation. Recently, treatment with FGF21 slowed the progression of obesity by downregulating ALT, AST and IL-6. ${ }^{34}$ There are recent reports that FGF21 attenuated CIA development, decreased inflammatory cytokine production and diminished the Th17-IL-17 axis via the STAT3 pathway. ${ }^{18,35}$ We observed that metformin reduced the levels of inflammatory mediators, such as IKBKE, which is a therapeutic target for obesityassociated inflammation ${ }^{36}$ and a means to increase FGF21 production in the liver and BAT. Therefore, our observations suggest that metformin has an anti-obesity effect, as well as an inflammatory effect, in obese CIA mice.

Previous research has documented the ability of metformin to induce FGF21 production, ${ }^{37}$ as well as attenuate the progression of obesity, thereby ameliorating dysregulated metabolic function. ${ }^{23,38,39}$ On the other hand, metformin decreases FGF21 expression. It has been suggested that treatment with metformin downregulates the FGF21 level in the plasma of type 2 diabetes mellitus patients. ${ }^{40,41}$ However, the effect of metformin on FGF21 levels is associated with an antiinflammatory effect. Metformin has also been shown to reduce the expression of high-sensitivity C-reactive protein, which is an inflammatory indicator that can demonstrate the progression of metabolic disorder in the plasma of type 2 diabetes mellitus patients. ${ }^{41}$ Metformin might decrease FGF21 levels by alleviating inflammation in type 2 diabetes mellitus patients.

Our findings demonstrate the possibility of metformin treatment for autoimmune arthritis associated with metabolic disease. Our study suggests that metformin ameliorates the 

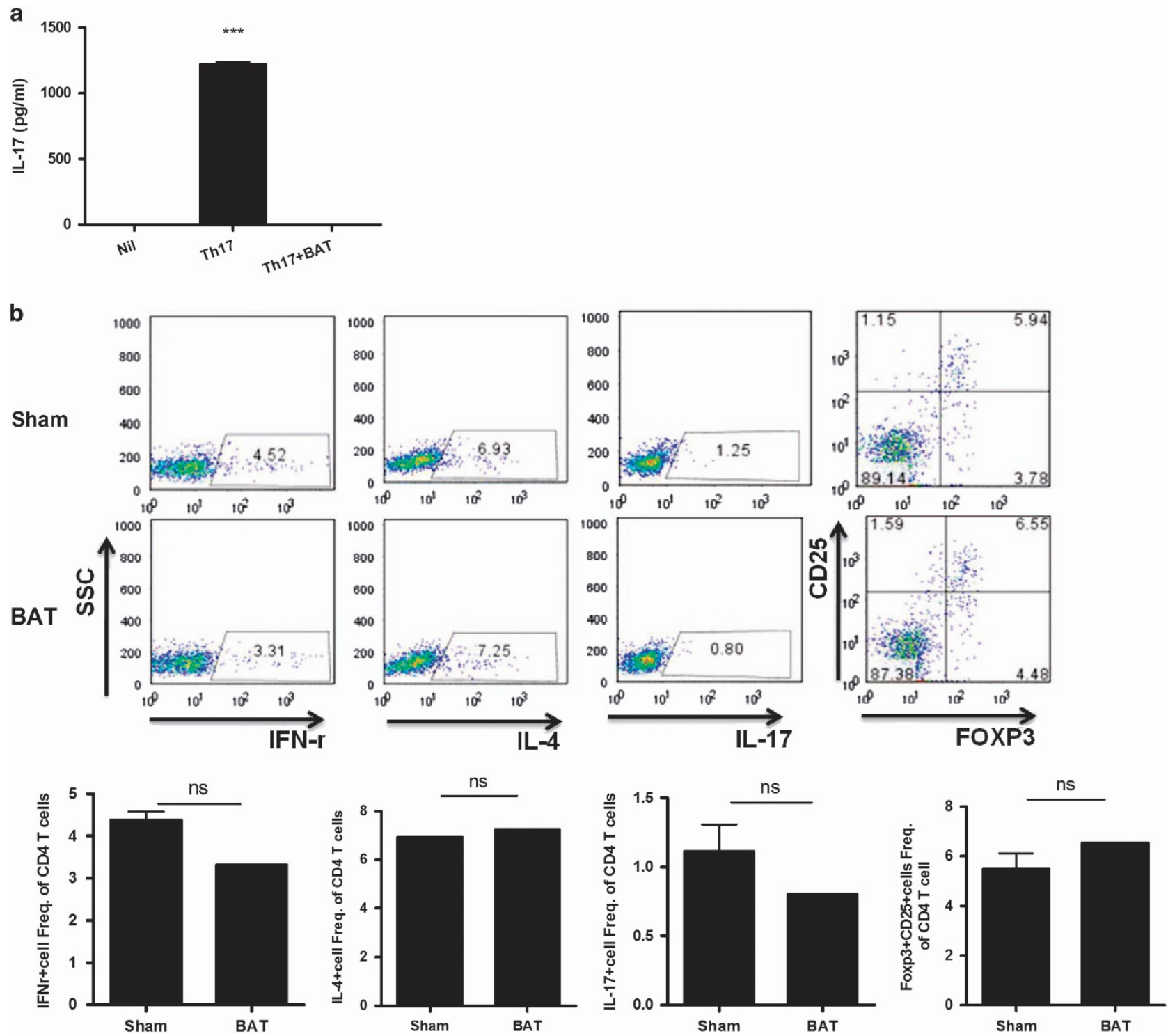

Figure 6 BAT reduced the activation of T cells and inhibited Th17 differentiation. (a) Splenocytes were cultured in Th17 conditions and co-cultured with BAT. IL-17 levels in the culture supernatants were measured by ELISA. (b) The differentiation of helper T cells and regulatory $T$ cells obtained from the spleens of mice that received BAT transplants.

inflammatory response and decreases obesity progression and may be a therapeutic candidate for RA associated with metabolic disorders.

\section{CONFLICT OF INTEREST}

The authors declare no conflict of interest.

\section{ACKNOWLEDGEMENTS}

This work was supported by the National Research Foundation of Korea (NRF) grant funded by the Korean government (MSIP) (NRF-2017R1A2B3007688). This research was supported by Basic Science Research Program through the National Research Foundation of Korea (NRF) funded by the Ministry of Education (NRF-2015R1D1A1A01058153). This research was supported by a grant from the Korea Health Technology R\&D Project through the Korea Health Industry Development Institute (KHIDI), funded by the
Ministry of Health \& Welfare, Republic of Korea (grant number: HI15C3062).

\section{PUBLISHER'S NOTE}

Springer Nature remains neutral with regard to jurisdictional claims in published maps and institutional affiliations.

1 Smolen JS, Redlich K, Zwerina J, Aletaha D, Steiner G, Schett G. Pro-inflammatory cytokines in rheumatoid arthritis: pathogenetic and therapeutic aspects. Clin Rev Allergy Immunol 2005; 28: 239-248.

2 Maini R, St Clair EW, Breedveld F, Furst D, Kalden J, Weisman M et al. Infliximab (chimeric anti-tumour necrosis factor alpha monoclonal antibody) versus placebo in rheumatoid arthritis patients receiving concomitant methotrexate: a randomised phase III trial. ATTRACT Study Group. Lancet 1999; 354: 1932-1939.

3 Finckh A, Simard JF, Gabay C, Guerne PA, for the physicians SCQM, Evidence for differential acquired drug resistance to anti-tumour necrosis factor agents in rheumatoid arthritis. Ann Rheum Dis 2006; 65: 746-752. 
4 Prajapati R, Plant D, Barton A. Genetic and genomic predictors of anti-TNF response. Pharmacogenomics 2011; 12: 1571-1585.

5 Hetland ML, Christensen IJ, Tarp U, Dreyer L, Hansen A, Hansen IT et al. Direct comparison of treatment responses, remission rates, and drug adherence in patients with rheumatoid arthritis treated with adalimumab, etanercept, or infliximab: results from eight years of surveillance of clinical practice in the nationwide Danish DANBIO registry. Arthritis Rheum 2010; 62: 22-32.

6 Paula FS, Alves JD. Non-tumor necrosis factor-based biologic therapies for rheumatoid arthritis: present, future, and insights into pathogenesis. Biologics 2014; 8: 1-12.

7 Thomson TM, Lescarbeau RM, Drubin DA, Laifenfeld D, de Graaf D, Fryburg DA et al. Blood-based identification of non-responders to anti-TNF therapy in rheumatoid arthritis. BMC Med Genomics 2015; 8: 26.

8 Manning PJ, Sutherland WH, McGrath MM, de Jong SA, Walker RJ, Williams MJ. Postprandial cytokine concentrations and meal composition in obese and lean women. Obesity 2008; 16: 2046-2052.

9 Patel C, Ghanim H, Ravishankar S, Sia CL, Viswanathan P, Mohanty P et al. Prolonged reactive oxygen species generation and nuclear factor-kappaB activation after a high-fat, high-carbohydrate meal in the obese. J Clin Endocrinol Metab 2007; 92: 4476-4479.

10 Haslam DW, James WP. Obesity. Lancet 2005; 366: 1197-1209.

11 Crowson CS, Matteson EL, Davis JM 3rd, Gabriel SE. Contribution of obesity to the rise in incidence of rheumatoid arthritis. Arthritis Care Res 2013; 65: 71-77.

12 Kwon $\mathrm{H}$, Pessin JE. Adipokines mediate inflammation and insulin resistance. Front Endocrinol 2013; 4: 71.

13 Harms M, Seale P. Brown and beige fat: development, function and therapeutic potential. Nat Med 2013; 19: 1252-1263.

14 Kharitonenkov A, Shiyanova TL, Koester A, Ford AM, Micanovic R, Galbreath EJ et al. FGF-21 as a novel metabolic regulator. J Clin Invest 2005; 115: 1627-1635.

15 Fisher FM, Chui PC, Antonellis PJ, Bina HA, Kharitonenkov A, Flier JS et al. Obesity is a fibroblast growth factor 21 (FGF21)-resistant state. Diabetes 2010; 59: 2781-2789.

16 Foltz IN, Hu S, King C, Wu X, Yang C, Wang W et al. Treating diabetes and obesity with an FGF21-mimetic antibody activating the betaKlotho/FGFR1c receptor complex. Sci Trans/ Med 2012; 4: 162ra153.

17 Hanssen MJ, Broeders E, Samms RJ, Vosselman MJ, van der Lans AA, Cheng CC et al. Serum FGF21 levels are associated with brown adipose tissue activity in humans. Sci Rep 2015; 5: 10275.

18 Yu Y, Li S, Liu Y, Tian G, Yuan Q, Bai F et al. Fibroblast growth factor 21 (FGF21) ameliorates collagen-induced arthritis through modulating oxidative stress and suppressing nuclear factor-kappa B pathway. Int Immunopharmacol 2015; 25: 74-82.

19 Campbell IW, Menzies DG, Chalmers J, McBain AM, Brown IR. One year comparative trial of metformin and glipizide in type 2 diabetes mellitus. Diabete Metab 1994; 20: 394-400.

20 Hallsten K, Virtanen KA, Lonnqvist F, Sipila H, Oksanen A, Viljanen T et al. Rosiglitazone but not metformin enhances insulin- and exercise-stimulated skeletal muscle glucose uptake in patients with newly diagnosed type 2 diabetes. Diabetes 2002; 51: 3479-3485.

21 Son HJ, Lee J, Lee SY, Kim EK, Park MJ, Kim KW et al. Metformin attenuates experimental autoimmune arthritis through reciprocal regulation of Th17/Treg balance and osteoclastogenesis. Mediators Inflamm 2014; 2014: 973986.

22 Lee SY, Lee SH, Yang EJ, Kim EK, Kim JK, Shin DY et al. Metformin ameliorates inflammatory bowel disease by suppression of the STAT3 signaling pathway and regulation of the between Th17/Treg balance. PLoS ONE 2015; 10: e0135858.

23 Kim EK, Lee SH, Jhun JY, Byun JK, Jeong JH, Lee S-Y et al. Metformin prevents fatty liver and improves balance of white/brown adipose in an obesity mouse model by inducing FGF21. Mediators Inflamm 2016; 2016: 13.

24 Jhun J, Lee S, Kim SY, Na HS, Kim EK, Kim JK et al. Combination therapy with metformin and coenzyme Q10 in murine experimental autoimmune arthritis. Immunopharmacol Immunotoxicol 2016; 38: 103-112.

25 Jhun J, Lee SH, Byun JK, Jeong JH, Kim EK, Lee J et al. Coenzyme Q10 suppresses Th17 cells and osteoclast differentiation and ameliorates experimental autoimmune arthritis mice. Immunol Lett 2015; 166: 92-102.
26 Gao M, Ma Y, Liu D. High-fat diet-induced adiposity, adipose inflammation, hepatic steatosis and hyperinsulinemia in outbred CD-1 mice. PLOS ONE 2015; 10: e0119784.

27 Lee MS, Kim IH, Kim CT, Kim Y. Reduction of body weight by dietary garlic is associated with an increase in uncoupling protein mRNA expression and activation of AMP-activated protein kinase in diet-induced obese mice. J Nutr 2011; 141: 1947-1953.

$28 \mathrm{Yu} \mathrm{H}$, Pardoll D, Jove R. STATs in cancer inflammation and immunity: a leading role for STAT3. Nat Rev Cancer 2009; 9: 798-809.

29 Shackelford DB, Shaw RJ. The LKB1-AMPK pathway: metabolism and growth control in tumour suppression. Nat Rev Cancer 2009; 9: 563-575.

30 Zhou J, Wulfkuhle J, Zhang H, Gu P, Yang Y, Deng J et al. Activation of the $\mathrm{PTEN} / \mathrm{mTOR} / \mathrm{STAT} 3$ pathway in breast cancer stem-like cells is required for viability and maintenance. Proc Natl Acad Sci USA 2007; 104: 16158-16163.

31 Madhok R, Crilly A, Watson J, Capell HA. Serum interleukin 6 levels in rheumatoid arthritis: correlations with clinical and laboratory indices of disease activity. Ann Rheum Dis 1993; 52: 232-234.

32 Sack U, Kinne RW, Marx T, Heppt P, Bender S, Emmrich F. Interleukin-6 in synovial fluid is closely associated with chronic synovitis in rheumatoid arthritis. Rheumatol Int 1993; 13: 45-51.

33 van Hamburg JP, Asmawidjaja PS, Davelaar N, Mus AM, Colin EM, Hazes JM et al. Th17 cells, but not Th1 cells, from patients with early rheumatoid arthritis are potent inducers of matrix metalloproteinases and proinflammatory cytokines upon synovial fibroblast interaction, including autocrine interleukin-17A production. Arthritis Rheum 2011; 63: 73-83.

34 Wang WF, Li SM, Ren GP, Zheng W, Lu YJ, Yu YH et al. Recombinant murine fibroblast growth factor 21 ameliorates obesity-related inflammation in monosodium glutamate-induced obesity rats. Endocrine 2015; 49 : $119-129$.

35 Li SM, Yu YH, Li L, Wang WF, Li DS. Treatment of CIA mice with FGF21 down-regulates TH17-IL-17 axis. Inflammation 2016; 39: 309-319.

36 Reilly SM, Chiang SH, Decker SJ, Chang L, Uhm M, Larsen MJ et al. An inhibitor of the protein kinases TBK1 and IKK-varepsilon improves obesity-related metabolic dysfunctions in mice. Nat Med 2013; 19: 313-321.

37 Nygaard EB, Vienberg SG, Orskov C, Hansen HS, Andersen B. Metformin stimulates FGF21 expression in primary hepatocytes. Exp Diabetes Res 2012; 2012: 465282.

38 Virtanen KA, Hallsten K, Parkkola R, Janatuinen T, Lonnqvist F, Viljanen T et al. Differential effects of rosiglitazone and metformin on adipose tissue distribution and glucose uptake in type 2 diabetic subjects. Diabetes 2003; 52: 283-290.

39 Baptista T, Rangel N, Fernandez V, Carrizo E, El Fakih Y, Uzcategui E et al. Metformin as an adjunctive treatment to control body weight and metabolic dysfunction during olanzapine administration: a multicentric, double-blind, placebo-controlled trial. Schizophr Res 2007; 93: 99-108.

40 Zhang M, Liu Y, Xiong ZY, Deng ZY, Song HL, An ZM. Changes of plasma fibroblast growth factor-21 (FGF-21) in oral glucose tolerance test and effects of metformin on FGF-21 levels in type 2 diabetes mellitus. Endokrynol Pol 2013; 64: 220-224.

41 Fan $\mathrm{H}$, Sun X, Zhang H, Liu J, Zhang P, Xu Y et al. Effect of metformin on fibroblast growth factor-21 levels in patients with newly diagnosed type 2 diabetes. Diabetes Technol Ther 2016; 18: 120-126.

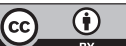

This work is licensed under a Creative Commons Attribution 4.0 International License. The images or other third party material in this article are included in the article's Creative Commons license, unless indicated otherwise in the credit line; if the material is not included under the Creative Commons license, users will need to obtain permission from the license holder to reproduce the material. To view a copy of this license, visit http:// creativecommons.org/licenses/by/4.0/

C) The Author(s) 2018 